\title{
Rapid assembly of planar quadrangular, self-interlocking modules to anticlastically curved forms
}

\author{
G. H. Filz \& S. Schiefer \\ Institute of Design | unit koge, Structure and Design, \\ Faculty of Architecture, University of Innsbruck, Austria
}

\begin{abstract}
Rapid assembly implies simple and fast fabrication as well as simple (archi-) tectonics enabled by simple or without joints. This paper presents preconditions and principles how planar quadrangular, self-interlocking modules can rapidly be assembled to self-supporting, anticlastically curved forms and focuses to minimal surfaces of catenoids. The rotationally symmetric shapes of catenoids can be panelized into quadrangular, planar modules, which again can be assembled from planar elements. Simultaneously self-interlocking behavior is guaranteed by module-geometry where - similar to reciprocal frame systems - angles of two opposite sidewalls are leaning in counter-wise directions. An additional guide and joint-elements are not necessary but could support simple and rapid assembly into larger spatial structures. Due to planarity and linear cuts small amounts of data are necessary for fabrication and assembly of industrially supplied sheet-elements. Following the above mentioned principles we can find various structurally efficient forms composed of one or more possibly also intersecting catenoids. The probably most interesting ones are those which can be rotated in space at will by keeping their structural performance and interlocking qualities. Our method allows for a high degree of customized solutions in shape and scale. Self-interlocking modules can easily be assembled to temporary/permanent structures or formwork, disassembled and reassembled to other customized forms or parts of new constellations. Ongoing investigations are dealing with possibilities and limits of forms, structural performance and on-purpose-manipulation of the sidewalls' angles of neighbored modules.

Keywords: self-interlocking modules, anticlastically curved minimal surface, catenoid, panelization.
\end{abstract}




\section{Context}

MARAS 2014 asks for mobile and rapidly assembled (temporary) structures and also points to the importance of speed of response directly connected to efficient design of assembly joints, reversible deployment and potential reuse. Seen as a process-chain, rapid assembly implies simple and fast fabrication as well as simple (archi-) tectonics enabled by simple or without joints.

This paper presents preconditions and principles how planar quadrangular, selfinterlocking modules can rapidly be assembled to structurally efficient, selfsupporting, anticlastically curved forms.

The emergence of the attributes planar quadrangular, self-interlocking and structurally efficient respectively self-supporting simultaneously can be seen as requirement, necessity and interdependence. This way we can define possibilities and limits of our method in a process of elimination.

For structural benefits caused by equally distributed radii of curvature and stresses of such forms this research focuses but is not limited to anticlastic minimal surfaces of catenoids in soap film analogy.

Catenoids are rotationally symmetric, anticlastically curved shapes that can be panelized by quadrangular meshing into planar, self-interlocking modules, which again can be assembled from planar elements. All necessary cuts are linear and planar too.

\section{Concepts for panelization spatially curved forms into planar, quadrangular elements}

In general we know that besides planes all synclastic and anticlastic surfaces that are the result of rotational symmetry, all translational surfaces deriving from sweeping any line or curve on one rail and some ruled surfaces can be subdivided into planar quadrangular elements. Of course all surfaces can be triangulated but the later on more precisely described requirement of self-interlocking can only be fulfilled by firstly anticlastically curved and secondly quadrangular subdivided surfaces. In this context material thickness is postulated.

Basically the two concepts of surface-based and mesh-based panelization were examined in approaching the issue of subdividing spatially curved forms in order to receive planar elements.

\subsection{Surface-based panelization}

In our research this approach was used to examine shapes of the minimal surfaces only. This means that the form (coalesce covering structural and geometrical aspects) and not its form generating (mesh) elements are subject of examination.

One possibility is to use the principles of dense packing (Figs 1-2) to generate patterns on the surface, which might lead to planar panelization. 

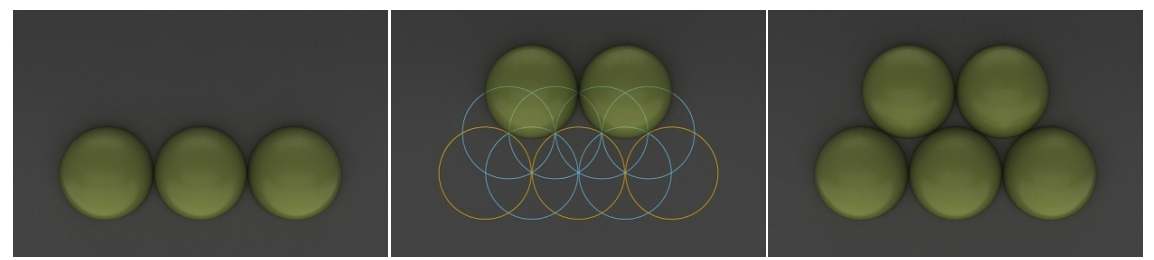

Figure 1: Development of dense packing with arranged identical spheres and intersected with a plane surface.
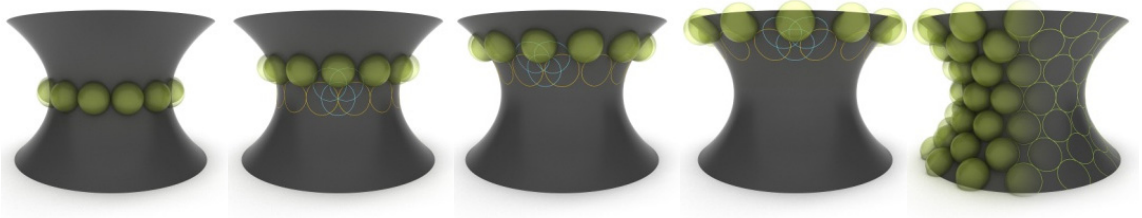

Figure 2: Geometry and arrangement of dense packed identical spheres with the middle row spheres having one tangent point with each neighbor on the surface of the catenoid.

\subsubsection{Dense packing of identical spheres on anticlastically curved minimal surfaces}

The intersection of dense packed identical spheres and surface shows gaps in the resulting spatially curved pattern except the middle row of the illustrated object. These spheres were arranged to have one tangent point with each neighbor on the surface. For this reason these spheres intersect inside the catenoid and have 6 tangent points with their neighbors. All other spheres have 4 tangent points (Fig. 2). Shifting the center of each sphere on the surface causes gaps between the middle row spheres already. This way all spheres are limited to 4 tangent points with their neighbors.

With this approach no solution - including the duals of the generated pattern delivering planar, quadrangular elements could be found.

\subsubsection{Non-identical curves from dense packing of non-identical spheres or mapping of circles on anticlastically curved minimal surfaces}

Another approach focuses on the subdivision of the shape by using geometrical methods like dense packing of non-identical spheres or mapping of plane elements onto the minimal surface.

With the method of mapping we obtain gap-free patterns but the formerly plane circles will be distorted and spatially curved (Fig. 3). The generated patterns and their duals, which are generated with above described method, lead to rough and therefore unsatisfying surface-approximation. 
When distributing spheres with different radii on the surface gaps disappear, but spatially curved intersection-lines in the pattern cannot be avoided. In this case this was examined by the Galapagos solver for Rhinoceros (Fig. 4).

Therefore neither the generated patterns nor their duals, which were generated with the above described method, led planar quadrangular elements.

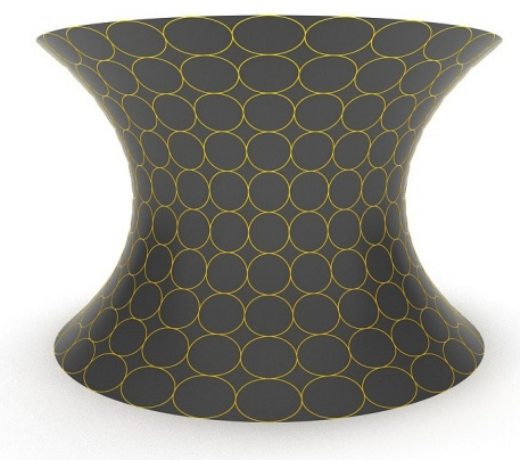

Figure 3: Mapping circles on the surface of the catenoid.

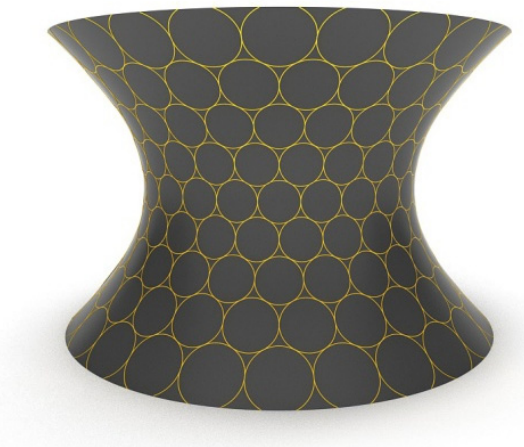

Figure 4: Distribution of circles on surface by Galapagossolver.

\subsection{Mesh-based panelization}

Most of the software that is used for formfinding and calculation of structural membranes is mesh-based. In the case of minimal surface catenoids all mesh-faces deriving from the structural equilibrium-mesh can be filled with planar surfaces. So this method covers the desire for anticlastically curved architectural spaces as well as the materialization with plane elements provided by the industry by keeping the structural and geometrical unity and for this reason spatial harmony and architectural aesthetics.

\subsubsection{Mesh-face patterning}

This approach is based on the direct link of pattern and form-generating mesh of the catenoid. The mesh is generated by Rhino Membrane. Grasshopper is used to generate different patterns in order to provide ideally distributed mesh-patterns for the generation of the minimal surface. This parallel procedure guarantees a direct correlation of the specifications of initial pattern and the generated "meshpattern". This way the result can be influenced or guided and it is directly related to the initially selected UV-values.

\subsubsection{Planar mesh-faces}

Mesh faces are planar for all rotational-symmetric forms. The verification of planarity is checked by a Rhinoceros/Grasshopper analysis with a tolerance of $1 / 100 \mathrm{~mm}$ per mesh face (Figs 5(a)-(c)). Deviations in this range were neglected as marginal. In general we can observe a low number of different mesh faces at 
rotational-symmetric forms and in special at rotational-symmetric minimal surfaces.

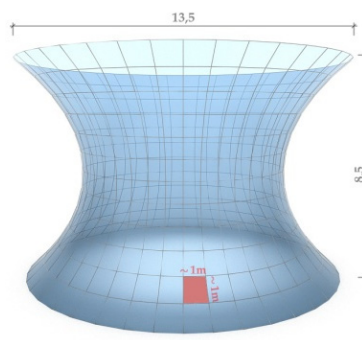

(a)

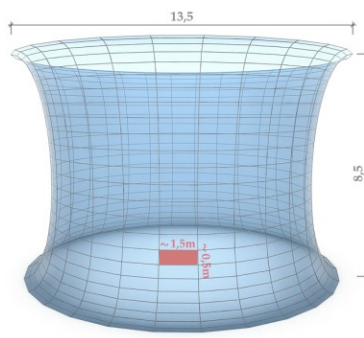

(b)

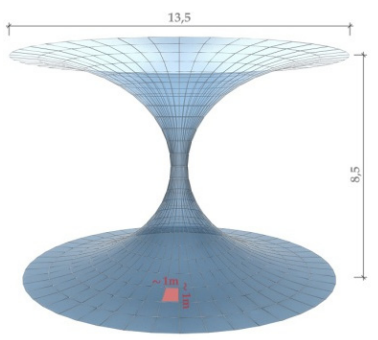

(c)

Figure 5: (a) Catenoid with planar faces; tolerance 1/100mm per mesh-face; (b) Other rotational-symmetric form with planar faces; tolerance $1 / 100 \mathrm{~mm}$ per mesh-face; (c) Other rotational-symmetric form with planar faces; tolerance $1 / 100 \mathrm{~mm}$ per mesh-face.
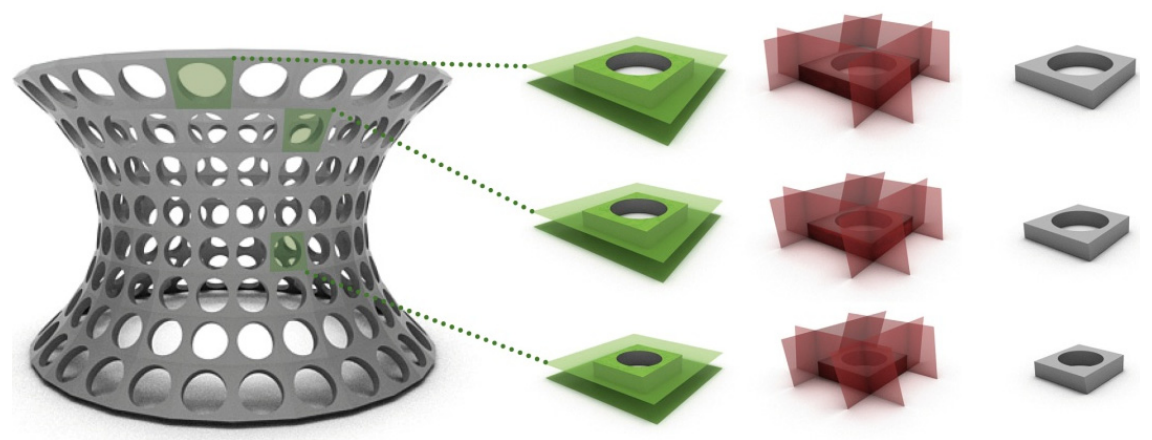

Figure 6: Rotational-symmetric catenoid assembled from planar elements with planar cuts.

Figure 6 shows that rotational-symmetric catenoids can be assembled from planar elements with planar cuts as a good basis for the fabrication of these elements. The complex spatially curved form of a minimal surface catenoid faces relatively small amounts of data and simple tools for its realization.

A deviation from rotational-symmetric bodies increases the necessary level of tolerance for planarity. Due to the balanced and uniform curvature of minimal surfaces, which have equal radii of main curvature in each point on its surface, this level of tolerance is lower compared to other anticlastic surfaces (Figs 7(a)-(c)). 

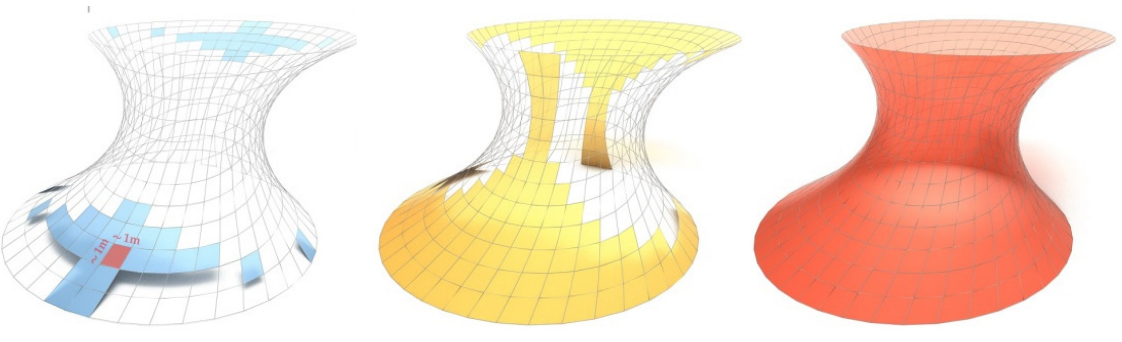

Figure 7: (a) Shifted catenoid; tolerance 0.1\%; (b) Shifted catenoid; tolerance $1 \%$; (c) Shifted catenoid; tolerance $10 \%$.

\section{Self-interlocking modules}

Mobility and rapid assembly of structures asks for simple joining-techniques like joining by stacking which possibly can be facilitated by guide elements.

Self-interlocking offers essential advantages in the assembling process and simultaneously there is no need of scaffolding. The structural performance of the stacked form also benefits from the self-interlocking behavior of assembled modules.

The self-interlocking behavior of our proposal can be gained by three parameters:

\subsection{Material thickness}

Material thickness is obtained by the offset of the anticlastically curved surface. The dimension of the surface-offset can freely be chosen. Basically we can increase self-interlocking behavior by increasing the value of the offset.

\subsection{Anticlastic curvature}

As we know, at anticlastically curved surfaces the circles of curvature are on opposite sides of the surface. Therefore - similar to reciprocal frame systems angles of two opposite sidewalls of each module are leaning in counter-wise directions. Caused by the overall geometry of catenoids all sidewalls are different from $90^{\circ}$.

In comparison to conventional reciprocal frame systems we receive forms without holes and/or excess length.

Self-interlocking modules from planes or synclastically curved surfaces can only be generated by on-purpose-manipulation of sidewalls' angles but gaps or holes between the modules cannot be avoided.

Subsequent increase of angles at anticlastically curved surfaces can lead to increased self-interlocking behavior but also causes gaps or holes between the modules. 


\subsection{Quadrangular modules}

As already mentioned above, quadrangular panelized modules are necessary in order to provide two pairs of counter-wisely leaning angles in addition to the fact of being planar for rotationally symmetric surfaces and in contrast to polygonal modules with more than four sidewalls.

Since the rule of two pairs of counter-wisely leaning angles is not fulfilled, triangulated modules are not self-interlocking.

\section{Structural and self-supporting behavior}

The shape of the catenoid is basically generated by a catenary that rotates around a longitudinal axis. Usually generated by two circular rings it is the only rotational body that can be minimal surface at the same time. [Otto IL18, 1987, S.112 and SFB 230/7 1992, S.165]

Together with the fact that soap film can bear uniform tension only we can assume that all other rotational forms have shear forces and moments. Caused by equally distributed radii of curvature and stresses of minimal surfaces/soapfilm, structural benefits can be expected from forms inverted from tension to compression. Therefore this research focuses but is not limited to anticlastic minimal surfaces of catenoids in soap film analogy.

Compared to other rotationally symmetrical forms the angles between the modules of panelized catenoids show equally and harmonic distribution.

Modules that are assembled from planar elements save self-weight. At the same time the sidewall-elements form load bearing, circular and catenary arches.

One of the probably most interesting aspects is that the self-interlocking behavior of the assembled forms is not limited to a special setup, configuration, constellation or position in space. In opposite, these forms can be rotated in space at will by keeping their structural performance and interlocking qualities.

\section{Fabrication: mass and customization.}

Our research addresses the desire for individually formed spaces as well as the standardized products of industry, which provides plane elements, easy to produce, easy to handle, easy to pile, easy to store, easy to transport and so on.

Seen as a process-chain, rapid assembly implies simple and fast fabrication as well as simple (archi-) tectonics enabled by simple or without joints.

Figures 8 and 9 show two intersected catenoids and their panelization into quadrangular planar modules. Three different modules are assembled from planar elements (Figs 10 and 11). All necessary cuts are linear and planar too. Fig. 12 illustrates the arrangement of all parts of the three modules on standardized wooden sheet-material, ready for fabrication.

Due to planarity and linear cuts small amounts of data are necessary for fabrication and assembly. 


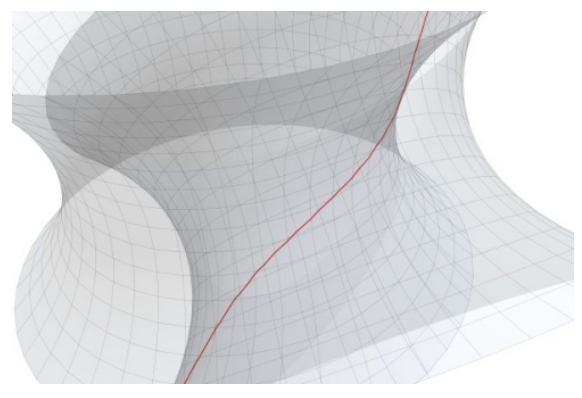

Figure 8: Intersection of two catenoids.

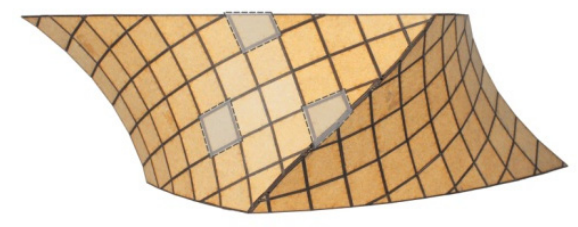

Figure 10: Selected cut-out and three representing modules.

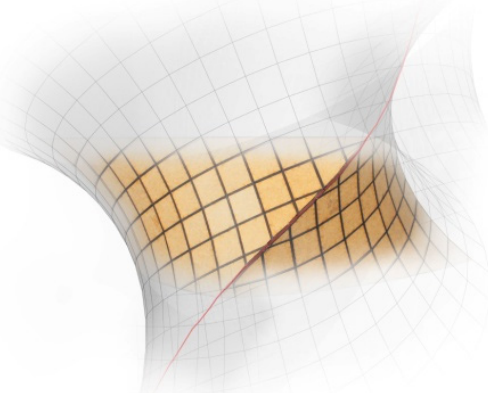

Figure 9: Overlay of digital and physical model.

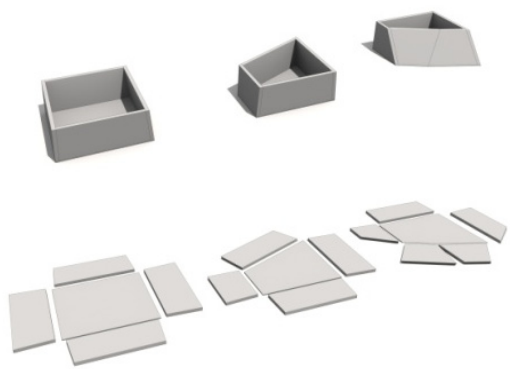

Figure 11: Representing modules and their elements.

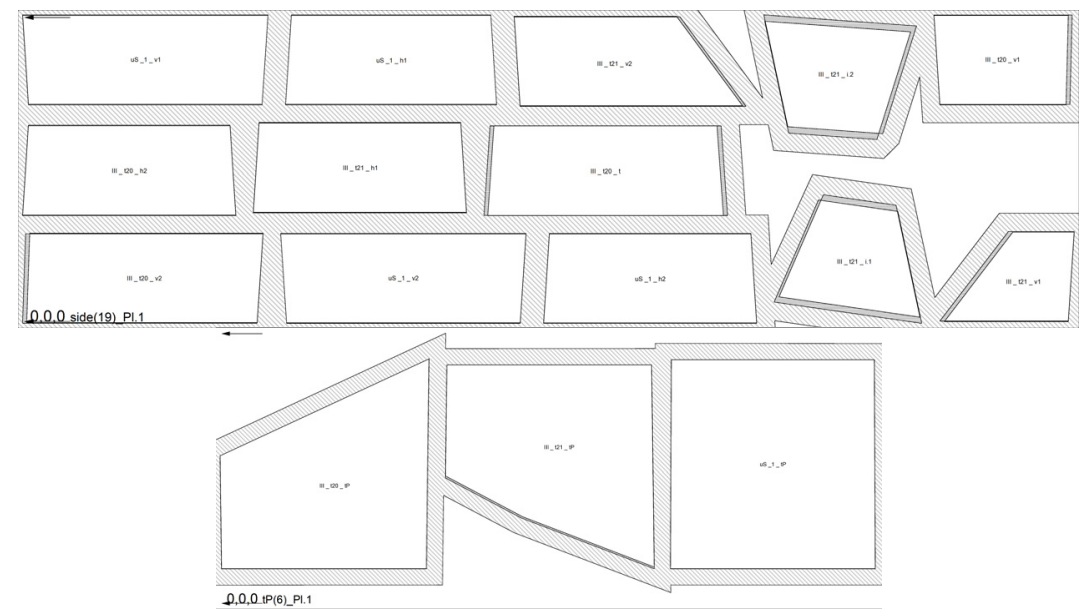

Figure 12: Arrangement of all parts of the three modules on standardized wooden sheet-material, ready for fabrication. 


\section{Anticlastically curved forms}

Following above mentioned principles we can find various structurally efficient forms and constellations composed of one or more possibly also intersecting catenoids.

Minimal surfaces as well as defined cut-outs from the surface have the property that they can be scaled and/or rotated in space at will by keeping their form and structural performance. This offers a whole variety of forms and spatial curvature.

First arrangements of two or more intersecting catenoids show architecturally interesting forms, whereas the intersection line, which is spatially curved too, and all truncated modules represent challenging geometrical tasks (Fig. 13).

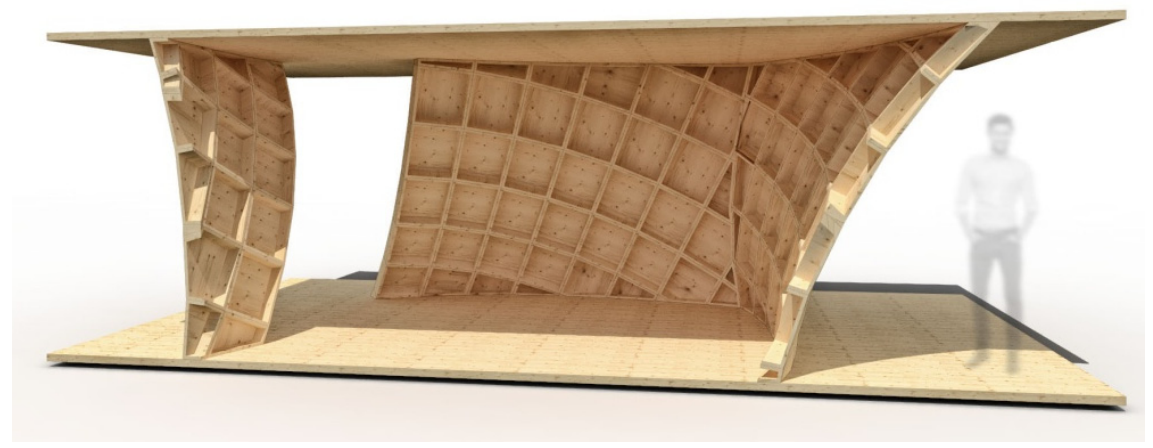

Figure 13: Visualization of 1/1 prototype - proof of concept.

\section{Conclusion and perspective}

Our method to panelize catenoids into quadrangular, planar modules allows for a high degree of customized solutions in shape and scale.

Self-interlocking modules can easily be assembled to temporary/permanent structures or formwork, disassembled and reassembled to other customized forms or parts of new constellations. Latest results in studies of forms, shows different types of intersections that have strong influence on the structural behavior of the overall form and that there are possibilities for frameless solutions and intersections.

Ongoing investigations are dealing with possibilities and limits of forms, structural performance and on-purpose-manipulation of the sidewalls' angles of neighbored modules.

\section{References}

[1] Otto, Frei, IL 18, Seifenblasen, Karl Krämer Verlag, Stuttgart 1988 Mitteilungen des SFB230, Heft7, Sonderforschungsbereich 230, Natürliche Konstruktionen - Leichtbau in Architektur und Natur, Universität Stuttgart, Universität Tübingen, Sprint Druck GmbH, Stuttgart 1992 
[2] Gaß, Siegfried, IL 25, Form, Form-Kraft-Masse 5, Experimente, Karl Krämer Verlag, Stuttgart 1990

[3] Bletzinger, Kai-Uwe, Kapitel "Differential Geometry and Continuum Mechanical Basics" in Numerical Theory, Lecture Notes, March 2009

[4] Bletzinger, Kai-Uwe, Kapitel "Introduction to Formfinding" in Numerical Theory, Lecture Notes, March 2009

[5] Pottmann, Helmut, Asperl, Andreas, Hofer, Michael, Kilian, Axel, Architectural Geometry, Bentley Institute Press, Exton, PA 19341, 2007

[6] Filz, Günther, Das Weiche Haus soft.spaces, Dissertation, LeopoldFranzens-Universität Innsbruck, Fakultät für Architektur, Juli 2010

[7] Filz, Günther (2011): minimal is maximal_soft spaces. In: International Association for Bridge and Structural Engineering; International Association for Shell and Spatial Structures: "Taller, Longer, Lighter. Meeting Growing Demand with Limited Resources”, 2011 IABSEIASS Symposium. Proceedings. London: Hemming Group, ISBN 978-07079-7122-3

[8] Filz, Günther , "soft.spaces _ new strategies for membrane architecture", International Conference on Textile Composites and Inflatable Structures, Structural Membranes 2011, CIMNE, Barcelona, Spain, 5-7 October 2011

[9] Filz, Günther H. (2012): Virtual and Physical Soft Spaces from Catenoids. In: Kim, Seung Deog: From Spatial Structures to Space Structures, IASSAPCS 2012. Abstract Book. Seoul: Korean Association for Spatial Structures, ISBN 978-89-968907-1-3, S. 137

[10] A.V. Dyskin, Y. Estrin, A.J. Kanel-Belov, E. Pasternak, A new principle in design of composite materials: reinforcement by interlocked elements, Composites Science and Technology 63 (2003) 483-491

[11] Filz, Günther H. (2013): Das weiche Haus soft.spaces. Antiklastische Minimalflächen als Elemente und Formensprache der Architektur. In: Schaur, Eda: Konstruktion und Gestaltung. Neue Formwelten für Architektur. Innsbruck: Innsbruck University Press (IUP) (Edited volume series), ISBN 978-3-902811-94-3, S. 92-127

[12] Baverel, O., Nooshin, H., and Kuroiwa, Y. 2004. Configuration processing of nexorades using genetic algorithms. Jour. of the Intl. Assoc. for Shell and Spatial Structures 45, 2, 99-108

[13] Bertin, V. 2001. Variations of lever beam structures. In Proceedings of Conference on Growth and Form: The Engineering of Nature, School of Architecture, University of Waterloo

[14] Brocato, M., and Mondardini, L. 2010. Geometric methods and computational mechanics for the design of stone domes based on Abeille's bond. In Advances in Architectural Geometry, Springer, 149-162

[15] Chilton, J. 2009. Development of timber reciprocal frame structures in the UK. In Proceedings of IASS Symposium 2009: Evolution and trends in design, analysis and construction of shell and spatial structures, 1877-1884

[16] Douthe, C., and Baverel, O. 2009. Design of nexorades or reciprocal frame systems with the dynamic relaxation method. Computers \& Structures 87, 21 (Nov.), 1296-1307 
[17] Eigensatz, M., Kilian, M., Schiftner, A., Mitra, N., Pottmann, H., and Pauly, M. 2010. Paneling architectural freeform surfaces. ACM Trans. on Graphics (SIGGRAPH) 29, 4, 45:1-45:10

[18] Parigi, D., and Pugnale, A. 2012. Three-dimensional reciprocal structures: morphology, concepts, generative rules. In IASS-APCS Proc. from spatial structures to space structures

[19] Filz, Günther H., Schiefer, Simon, Stecher, Thomas (2013): Surface- and Mesh-based Approaches towards a Materialization of Architectural Catenoids. In: ICSA 2013: The 2nd International Conference on Structures and Architecture. Guimaraes, Portugal, 24.-26.07.2013. Guimaraes: ICSA 